\title{
Decomposição de palha de cana-de-açúcar recolhida em diferentes níveis após a colheita mecânica
}

\author{
Nilza Patrícia Ramos ${ }^{(1)}$, Carina Sayuri Yamaguchi(2), Adriana Marlene Moreno Pires ${ }^{(1)}$, \\ Raffaella Rossetto ${ }^{(3)}$, Rosana Aparecida Possenti( ${ }^{(4)}$, Ana Paula Packer ${ }^{(1)}$, \\ Osvaldo Machado Rodrigues Cabral ${ }^{(1)}$ e Cristiano Alberto de Andrade ${ }^{(1)}$
}

\begin{abstract}
(1)Embrapa Meio Ambiente, Caixa Postal 69, Rodovia SP 310, Km 127,5, CEP 13820-000 Jaguariúna, SP, Brasil. E-mail: nilza.ramos@embrapa.br,adriana.pires@embrapa.br,paula.packer@embrapa.br,osvaldo.cabral@embrapa.br,cristiano.andrade@embrapa.br (2)Instituto Agronômico, Avenida Barão de Itapura, o⒈481, Caixa Postal 28, CEP 13012-970 Campinas, SP, Brasil. E-mail: carinasy@gmail.com ${ }^{(3)}$ Agência Paulista de Tecnologia dos Agronegócios, Caixa Postal 28, CEP 13400-970 Piracicaba, SP, Brasil. E-mail: raffaella@apta.sp.gov.br (4)Instituto de Zootecnia, Rua Heitor Penteado, no 56, Centro, CEP 13460-000 Nova Odessa, SP, Brasil. E-mail: possenti@iz.sp.gov.br
\end{abstract}

Resumo - O objetivo deste trabalho foi determinar o efeito do recolhimento de quantidades variáveis da palha de cana-de-açúcar sobre sua decomposição na superfície do solo, após subsequentes socas. O experimento foi realizado por duas socas subsequentes, com a variedade de cana-de-açúcar RB-845210, tendo-se testado quatro quantidades remanescentes de palha após a colheita: 11,3, 8,5, 5,7 e 2,8 $\mathrm{Mg} \mathrm{ha}^{-1}$. Foram avaliadas as taxas de decomposição de biomassa, carbono, nitrogênio, celulose, hemicelulose e lignina, bem como a relação $\mathrm{C} / \mathrm{N}$ e a quantidade de $\mathrm{C}$ e $\mathrm{N}$ mineralizada a partir da palha. O recolhimento variável da palha da canade-açúcar não alterou as taxas de decomposição da biomassa nem a mineralização de carbono, hemicelulose, celulose e lignina, em uma mesma soca. O processo de decomposição da palha não se esgota, mesmo após duas socas, independentemente da quantidade inicial de resíduo sobre o solo. A relação $\mathrm{C} / \mathrm{N}$ e a decomposição da lignina servem como indicadores para verificar diferenças nas taxas de decomposição entre os níveis de palha avaliados, enquanto a decomposição da hemicelulose e da celulose somente detecta alterações nessas taxas ao longo do tempo. Em termos absolutos, quanto menor a retirada da palha do campo, maior a quantidade de carbono e nitrogênio mineralizada, mesmo que não haja diferenças na taxa de decomposição.

Termos para indexação: Saccharum, estoque de carbono, mineralização do nitrogênio, qualidade do solo, relação $\mathrm{C} / \mathrm{N}$, taxa de decomposição.

\section{Decomposition of sugarcane straw collected at different rates after mechanical harvest}

\begin{abstract}
The objective of this work was to determine the effect of collecting different levels of sugarcane straw on its decomposition on soil surface, after consecutive ratoons. The trial was conducted for two consecutive ratoons, using the sugarcane variety RB845210, testing four amounts of remaining straw after harvest: 11.3, 8.5, 5.7, and $2.8 \mathrm{Mg} \mathrm{ha}^{-1}$. Decomposition rates of biomass, carbon, nitrogen, cellulose, hemicellulose, and lignin were evaluated, as well as the $\mathrm{C} / \mathrm{N}$ ratio and the mineralized quantities of $\mathrm{C}$ and $\mathrm{N}$ from the straw. The distinct levels of sugarcane straw aboveground do not alter decomposition rates, neither do they alter the mineralization of carbon, hemicellulose, cellulose, and lignin within the same ratoon. The straw decomposition process persists along the two ratoon cycles, regardless of the initial amount of straw on soil surface. The $\mathrm{C} / \mathrm{N}$ ratio and lignin decay are adequate indicators for detecting differences of straw decomposition rates between the evaluated levels of straw, while hemicellulose and cellulose decay detects only differences in rates over time. In absolute terms, the lower the initial aboveground straw level, the higher the amounts of carbon and nitrogen mineralized, even if there are no differences in decomposition rates.
\end{abstract}

Index terms: Saccharum, carbon storage, nitrogen mineralization, soil quality, $\mathrm{C} / \mathrm{N}$ ratio, decomposition rate.

\section{Introdução}

O Brasil consolidou-se como protagonista mundial no cultivo da cana-de-açúcar (Saccharum spp. L.), com 721 milhões de toneladas de colmos produzidas em 2012, que superam em mais de 50\% a produção da Índia, segunda colocada neste tipo de exploração (Food..., 2014). O amplo aproveitamento da espécie na produção de açúcar e etanol, bem como no aproveitamento de resíduos para a produção de 
energia, ou para reciclagem de nutrientes para a própria cultura (Leite, 2009), incentiva a constante evolução dos sistemas de produção, com destacado foco na sustentabilidade da atividade (Rossetto et al., 2008).

A conversão da área com sistema de colheita com queima para colheita com cana-crua (sem despalha a fogo) tem sido proposta para reduzir as emissões de gases de efeito estufa e o trabalho manual desgastante (Leite, 2009). Essa conversão tem se concretizado especialmente após as inovações na área de mecanização da colheita, que têm possibilitado a deposição de mais de $20 \mathrm{Mg} \mathrm{ha}^{-1}$ ano $^{-1}$ de matéria seca de palha - folhas secas, ponteiro e restos de colmo sobre o solo (Vitti et al., 2008).

Atualmente, discute-se a possibilidade de recolher parcialmente a palha com vistas ao seu aproveitamento energético, seja elétrico ou para obtenção de etanol de segunda geração (Leal et al., 2013). Esta remoção, no entanto, pode alterar o sistema solo-planta-atmosfera envolvido no cultivo da cana-de-açúcar, por constituir uma situação intermediária entre a cana-queimada, que se desenvolve em solo sem cobertura vegetal, e a cana-crua, cuja palha permanece na superfície do solo. Portanto, a pesquisa tem se dedicado ao esclarecimento dos efeitos que a retirada da palha pode ocasionar no solo, especialmente quanto aos processos de decomposição.

Quanto à manutenção total da palha (cana-crua), sabe-se que ela é benéfica à proteção do solo, à infiltração e ao armazenamento de água, bem como à ciclagem de nutrientes (Peres et al., 2010; Sousa et al., 2012; Trivelin et al., 2013). A respeito da ciclagem, há variações na liberação de nutrientes pela decomposição, o que, por sua vez, é influenciado pelas condições edafoclimáticas e pela qualidade (nutrientes, celulose, hemicelulose e lignina) do resíduo depositado (Cotrufo et al., 2009). Assim, temperatura e umidade elevadas tendem a aumentar as taxas de decomposição de resíduos, enquanto elevadas relações $\mathrm{C} / \mathrm{N}(>100)$ e altas quantidades de lignina as reduzem (Melillo et al., 1982).

O estabelecimento de indicadores que possam representar mais significativamente o processo de decomposição ainda é um desafio para a pesquisa. Além do conteúdo químico, ou nutricional (teores de carbono, macro e micronutrientes), a literatura tem apontado o conteúdo bioquímico, ou estrutural (celulose, hemicelulose, lignina e polifenóis), como indicador seguro do processo de decomposição. Thomas \&
Asakawa (1993) e Zhang et al. (2008) concordam que a relação $\mathrm{C} / \mathrm{N}$ e o total de nutrientes contido na biomassa remanescente são os controladores do processo de decomposição da palha em escala global, e respondem por 39 e $31 \%$ das previsões de decomposição com modelos de simulações, respectivamente. Assim, eles podem ser utilizados para identificar o estágio do processo de decomposição e para decidir quanto aos ganhos ou perdas que a retirada da palha pode trazer ao sistema solo-planta-atmosfera.

Vitti et al. (2008) reportaram a importância que a adubação nitrogenada da cana pode ter sobre a relação $\mathrm{C} / \mathrm{N}$, o processo de decomposição e a liberação de $\mathrm{N}$ para o solo. Fortes et al. (2012) relataram a liberação de $93 \%$ do $\mathrm{N}$ de resíduos culturais da cana-deaçúcar, quando a relação $\mathrm{C} / \mathrm{N}$ destes chegou a 24 , e mineralização de 60,29 e $70 \%$ da lignina, celulose e hemicelulose, respectivamente. Com o uso de modelos de simulação, Trivelin et al. (2013) verificaram que o nitrogênio disponibilizado diminui com o recolhimento parcial da palha, ao longo de um ciclo (cinco cortes) de cana-de-açúcar. Simulações realizadas por Galdos et al. (2010), com cana-crua, revelaram incrementos no estoque de carbono no solo, a longo prazo, mas também que esses incrementos poderiam diminuir sob a condição de retirada parcial do resíduo.

Abramo Filho (1995), pioneiro no estudo da decomposição da palha da cana, relatou $60 \%$ de decomposição de biomassa, após uma soca, com a manutenção de $15 \mathrm{Mg} \mathrm{ha}^{-1}$ do resíduo sobre o solo. Aquino (2012) também observou taxa de decomposição próxima a $60 \%$, com a manutenção de $15 \mathrm{Mg} \mathrm{ha}^{-1} \mathrm{de}$ palha sobre o solo, significativamente inferior à taxa de $80 \%$ observada com manutenção de $20 \mathrm{Mg} \mathrm{ha}^{-1}$. Contudo, Ivo et al. (2013) não verificaram diferenças entre as taxas de decomposição da palha sob diferentes níveis de retirada parcial, o que reforça a necessidade de mais estudos para melhor entendimento dos efeitos do recolhimento.

O objetivo deste trabalho foi determinar o efeito do recolhimento de quantidades variáveis da palha de cana-de-açúcar sobre sua decomposição na superfície do solo, após subsequentes socas.

\section{Material e Métodos}

A pesquisa foi realizada em área experimental,

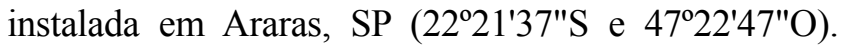
$\mathrm{O}$ canavial estudado vinha sendo cultivado com a

Pesq. agropec. bras., Brasília, v.51, n.9, p.1492-1500, set. 2016 DOI: 10.1590/S0100-204X2016000900048 
variedade RB-845210 sob sistema de cana-crua há mais de 10 anos e, durante as safras avaliadas de 2011/2012 e $2012 / 2013$, ele se encontrava em terceira e quarta soca. O solo da área foi classificado como Latossolo VermelhoAmarelo (Santos et al., 2006), com declividade inferior a $4 \%$. Na caracterização feita antes da terceira soca, ele apresentou $770 \mathrm{~g} \mathrm{~kg}^{-1}$ de argila; $39 \mathrm{~g} \mathrm{dm}^{-3}$ de matéria orgânica; $24 \mathrm{mg} \mathrm{dm}^{-3}$ de fósforo; além de 4, 46 e $23 \mathrm{mmol}_{\mathrm{c}}$ $\mathrm{dm}^{-3}$ de $\mathrm{K}, \mathrm{Ca}$ e $\mathrm{Mg}$, respectivamente; $73 \mathrm{mmol}_{\mathrm{c}} \mathrm{dm}^{-3}$ de CTC e $59 \%$ de saturação por bases (V). O clima do local, segundo classificação de Köppen, é do tipo Cwa: tropical úmido, com chuvas no verão e inverno seco (Rolim et al., 2007). Os dados de precipitação do período são apresentados na Figura 1.

Os tratamentos foram aplicados em setembro de 2011, imediatamente após a colheita mecanizada da cana-de-açúcar, tendo-se avaliado, nas duas socas, as quantidades de palha remanescente sobre o solo de $11,3,8,5,5,7$ e $2,8 \mathrm{Mg} \mathrm{ha}^{-1}$, que corresponderam, respectivamente, à manutenção em campo de 100, 75, 50 e $25 \%$ da palha (folhas secas, ponteiros e restos de colmo) produzida. A palha foi espalhada em toda a parcela, e não apenas acima dos sacos de decomposição acompanhados no estudo. A composição química inicial da palha foi de $47 \%$ de C, $0,47 \%$ de N, $36 \%$ de hemicelulose, $39 \%$ de celulose, $10 \%$ de lignina e relação $\mathrm{C} / \mathrm{N}$ de 100 .

As parcelas foram compostas por oito linhas de $15 \mathrm{~m}$ de comprimento, com espaçamento entre linhas de $1,5 \mathrm{~m}$, o que resultou em uma área total de $180 \mathrm{~m}^{2}$, com $117 \mathrm{~m}^{2}$ de área útil. Utilizou-se o delineamento experimental de blocos ao acaso, com parcelas subdivididas no tempo (socas) e quatro repetições. Os tratos culturais das duas socas seguiram a rotina da fazenda, com a adubação de cobertura realizada sem movimentação da palha, na quantidade de $100 \mathrm{~kg} \mathrm{ha}^{-1}$ de $\mathrm{N}$ e $150 \mathrm{~kg} \mathrm{ha}^{-1}$ de $\mathrm{K}_{2} \mathrm{O}$, com as fontes nitrato de amônio e cloreto de potássio. Não houve irrigação e nem aplicação de vinhaça ou de torta de filtro.

No estudo de decomposição, a palha retirada das parcelas foi secada à sombra, por 72 horas, homogeneizada e pesada em quantidades

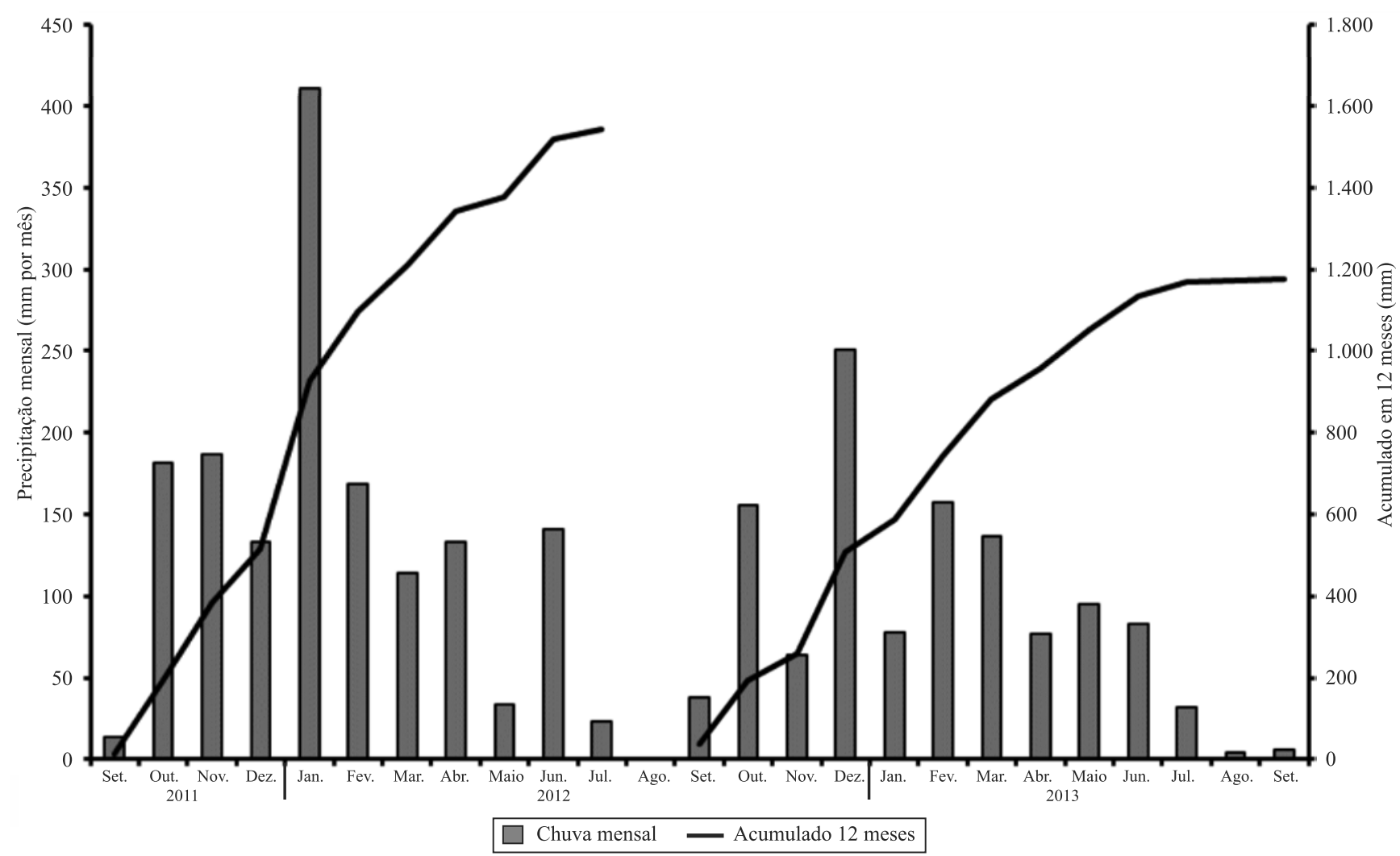

Figura 1. Precipitação pluviométrica durante as socas 2011/2012 ( $3^{\mathrm{a}}$ soca) e 2012/2013 (4 ${ }^{\mathrm{a}}$ soca), em área experimental com recolhimento variável de palha de cana-de-açúcar após a colheita. 
correspondentes aos tratamentos, para então ser depositada nos sacos de decomposição, seguindo metodologia adaptada de Bocock \& Gilbert (1957). Neste estudo, os sacos foram confeccionados em material plástico, nas dimensões $0,75 \times 0,50 \mathrm{~m}$ (malha de $15 \mathrm{~mm}$ ), maiores do que as indicadas no método original. Essa alteração foi proposta por Fortes et al. (2012), com a justificativa de ocupar o espaço desde a linha de plantio da cana-de-açúcar até metade da entrelinha, o que representaria melhor a condição natural de campo. Os tratamentos foram aplicados em agosto de 2011, e as coletas foram feitas antes das colheitas da $3^{\text {a }}$ (em julho de 2012, aos 281 da instalação do experimento) e da $4^{\mathrm{a}}$ socas (350 dias após a primeira avaliação). No momento das coletas, a palha foi retirada e transportada com cuidado rigoroso, a fim de evitar perdas e contaminações. As amostras seguiram para limpeza e secagem em estufa com circulação forçada de ar a $65^{\circ} \mathrm{C}$, até peso constante.

A determinação da massa de matéria seca $(\mathrm{g})$ foi feita em balança com duas casas decimais. Foram determinadas a biomassa de palha degradada ( $\mathrm{Mg} \mathrm{ha}^{-1}$ base seca), a partir da subtração da quantidade amostrada da biomassa remanescente, do valor inicial depositado nos tratamentos. Calculou-se então a taxa de degradação da biomassa (\%).

As amostras seguiram para moagem em moinho do tipo Willey, peneira 30 mesh do modelo Tecnal TE-650, e, posteriormente, para as análises dos teores (\%) de carbono e nitrogênio totais, pelo método de combustão por via seca, em analisador elementar de carbono e nitrogênio (TruSpec CN Leco). Assim como para a biomassa, foram calculadas as quantidades (Mg ha ${ }^{-1}$ base seca) e taxas (\%) de mineralização do carbono e nitrogênio, após cada soca. Também foi calculada a relação $\mathrm{C} / \mathrm{N}$ nos resíduos.

As determinações de celulose, hemicelulose e lignina (\%) foram realizadas no Laboratório de Análises Bromatológicas e Minerais do Instituto de Zootecnia, da Agência Paulista de Tecnologia dos Agronegócios, pelo método de Van Soest et al. (1991). Os teores (\%) de hemicelulose foram obtidos pela diferença entre o FDN (fibra em detergente neutro, ou conteúdo celular) e o FDA (fibra em detergente ácido, ou fração insolúvel estrutural). As taxas de decomposição (\%) de celulose e lignina foram determinadas seguindo o mesmo procedimento utilizado para $\mathrm{C}$ e $\mathrm{N}$.
Os resultados foram submetidos a análises de variância e regressão, tendo-se utilizado o teste de Tukey, a 1\% de probabilidade, para comparações entre as duas socas.

\section{Resultados e Discussão}

Houve interação significativa entre a quantidade de palha nos tratamentos e a soca avaliada, tanto para as taxas de mineralização da biomassa como do nitrogênio. Já a taxa de mineralização do carbono somente foi afetada pelas socas. $\mathrm{Na} 3^{\mathrm{a}}$ soca, a decomposição da biomassa foi de $65,5 \%$, sem diferenças entre as quantidades de palha avaliadas, que somente tiveram efeito sobre essa variável na $4^{\mathrm{a}}$ soca, com comportamento quadrático altamente significativo (Tabela 1). Ao final das duas socas, observou-se 90,7\% de decomposição da palhada.

Condições favoráveis de precipitação (Figura 1), aliadas à alta disponibilidade de nitrogênio, resultante da adubação de cobertura, possivelmente favoreceram a decomposição da biomassa na $3^{\mathrm{a}}$ soca. Assim, tanto o tratamento com manutenção da palha total (100\%) quanto o com manutenção de apenas $25 \%$ apresentaram substancial decomposição (65,5\% em média) ao final dessa soca. Também Ivo et al. (2013), ao estudarem quatro níveis de palha remanescente, não encontraram diferenças nas taxas de decomposição (média de $64 \%$ ) após a primeira soca, em Alagoas. Contudo, Aquino (2012) verificou diferenças de decomposição de até $30 \%$, ao comparar o menor nível de palha remanescente (5 $\mathrm{Mg} \mathrm{ha}^{-1}$ ) com o maior (22 $\mathrm{Mg} \mathrm{ha}^{-1}$ ), que decaiu $80 \%$ ao longo de uma soca, no Paraná. A compilação desses resultados, associados aos do presente estudo, permite inferir que taxas acima de $60 \%$ de decomposição de palha são esperadas dentro de uma soca de cana.

A taxa de mineralização do nitrogênio (Tabela 1) apresentou comportamento linear na $3^{\mathrm{a}}$ soca, com decréscimo dos valores em função do aumento da quantidade de palha remanescente, o que provavelmente indica que há imobilização deste elemento nos maiores níveis de palha. Já a taxa de mineralização do carbono (Tabela 1), assim como a da biomassa, não se diferenciou entre os níveis de palha, com média de $70,5 \%$, na $3^{\mathrm{a}}$ soca. Esses resultados comprovam que, para manter as mesmas taxas de mineralização do C e decomposição da biomassa, os tratamentos com maior quantidade de palha (75 e 100\%) imobilizam o 
$\mathrm{N}$ proveniente do solo ou dos fertilizantes, destinado ao suprimento da atividade microbiana, com possíveis reflexos negativos em sua disponibilidade para a planta (Vitti et al., 2008).

$\mathrm{Na} 4^{\mathrm{a}}$ soca, tanto a decomposição da biomassa quanto a mineralização do nitrogênio seguiram modelo quadrático (Tabela 1), com os menores valores obtidos próximos do tratamento com $50 \%$ de palha remanescente. A menor mineralização do $\mathrm{N}$ neste nível de palha explica a menor decomposição tanto da biomassa como da lignina (Tabela 2). O comportamento quadrático talvez se deva à redução na quantidade de substrato, no tratamento com $25 \%$, e a decomposição mais acelerada nos com 75 e $100 \%$, que possivelmente mativeram maiores níveis de umidade e possibilitaram atividade microbiana mais intensa. Observou-se aumento na mineralização do $\mathrm{N}$ da $3^{\mathrm{a}}$ para a $4^{\mathrm{a}}$ soca, nos tratamentos com mais palha $(100 \%$ e $75 \%)$, a qual passou de 18,9 para $56 \%$. Portanto, é provável que as elevadas taxas de mineralização do $\mathrm{C}$ tenham imobilizado $\mathrm{N}$, na $3^{\mathrm{a}}$ soca, e favorecido a sua liberação na $4^{\mathrm{a}}$ soca. Entretanto, estes valores foram inferiores aos observados por Fortes et al. (2012) que, em estudo sem remoção de palha, observaram mineralização de $68 \%$ do N na primeira soca e $93 \%$ na segunda. Já Vitti et al. (2008) relatam menores mineralizações do N, com valores entre 3 e $30 \%$. Os autores atribuem essas diferenças a variações nas condições edafoclimáticas e na composição inicial dos resíduos.

Observou-se diminuição linear da relação $\mathrm{C} / \mathrm{N}$ com o aumento no nível de palha remanescente sobre o solo, na $3^{\text {a }}$ soca (Figura 2), o que confirma os resultados de decomposição mais acelerada nos tratamentos com mais palha, que apresentaram elevadas taxas de

Tabela 1. Média \pm desvio-padrão das taxas de decomposição da biomassa e de mineralização do carbono e nitrogênio da palha, após a $3^{\mathrm{a}}$ e $4^{\mathrm{a}}$ soca, e após o período acumulado das duas socas (AC), em área com recolhimento variável de palha de cana-de-açúcar ${ }^{(1)}$.

\begin{tabular}{|c|c|c|c|c|c|c|c|c|c|}
\hline \multirow[t]{2}{*}{ Tratamento $^{(2)}$} & \multicolumn{3}{|c|}{ Taxa de decomposição da biomassa (\%) } & \multicolumn{3}{|c|}{ Taxa de mineralização do carbono (\%) } & \multicolumn{3}{|c|}{ Taxa de mineralização do nitrogênio (\%) } \\
\hline & $3^{\mathrm{a}}$ soca & $4^{\mathrm{a}}$ soca & Acumulado & $3^{\mathrm{a}}$ soca & $4^{\mathrm{a}}$ soca & Acumulado & $3^{\mathrm{a}}$ soca & $4^{\mathrm{a}}$ soca & Acumulado \\
\hline $100 \%$ & $65,0 \pm 0,7$ & $26,6 \pm 0,4$ & $91,6 \pm 0,9$ & $70,2 \pm 1,6$ & $24,5 \pm 1,7$ & $94,8 \pm 0,6$ & $18,9 \pm 2,7$ & $56,7 \pm 2,8$ & $75,7 \pm 2,7$ \\
\hline $75 \%$ & $66,4 \pm 3,2$ & $25,4 \pm 3,0$ & $91,7 \pm 0,5$ & $70,0 \pm 3,3$ & $24,8 \pm 2,9$ & $94,9 \pm 0,6$ & $18,9 \pm 6,6$ & $56,1 \pm 6,3$ & $75,1 \pm 2,5$ \\
\hline $50 \%$ & $67,9 \pm 3,6$ & $19,6 \pm 5,6$ & $87,5 \pm 2,0$ & $72,3 \pm 3,8$ & $20,8 \pm 5,3$ & $93,1 \pm 1,8$ & $34,1 \pm 4,9$ & $30,6 \pm 12,5$ & $66,7 \pm 7,6$ \\
\hline $25 \%$ & $62,9 \pm 2,7$ & $29,2 \pm 4,5$ & $92,1 \pm 1,9$ & $69,4 \pm 3,1$ & $27,0 \pm 3,8$ & $96,5 \pm 0,8$ & $36,1 \pm 8,2$ & $48,0 \pm 12,6$ & $82,2 \pm 4,8$ \\
\hline Média & $65,5 \mathrm{~A}$ & $25,2 \mathrm{~B}$ & 90,7 & $70,5 \mathrm{~A}$ & $24,3 \mathrm{~B}$ & 94,8 & $27,0 \mathrm{~B}$ & $47,9 \mathrm{~A}$ & 74,9 \\
\hline Regressão & ns & $* *(3)$ & ns & ns & ns & ns & $* *(4)$ & $* *(5)$ & ns \\
\hline CV (\%) & 3,38 & 12,84 & 1,66 & 3,68 & 12,57 & 1,06 & 20,71 & 19,48 & 6,5 \\
\hline
\end{tabular}

${ }^{(1)}$ Médias seguidas de letras iguais não diferem pelo teste de Tukey a $1 \%$ de probabilidade. ${ }^{(2)}$ Os tratamentos indicam a percentagem de palha que foi mantida no solo após a colheita: $100 \%, 11,3 \mathrm{Mg} \mathrm{ha}^{-1}$ de palha; $75 \%, 8,5 \mathrm{Mg} \mathrm{ha}^{-1} ; 50 \%, 5,7 \mathrm{Mg} \mathrm{ha}^{-1}$; e 25\%, 2,8 Mg ha ${ }^{-1}$. ns Não significativo. **Significativo a $1 \%$ de probabilidade. ${ }^{(3)} y=39,26-0,549 x+0,004 x^{2}, R^{2}=0,60 * * .{ }^{(4)} y=42,76-0,25 x, R^{2}=0,60 * * .{ }^{(5)} y=57,45-0,69 x+0,007 x^{2}, R^{2}=0,48 * *$.

Tabela 2. Média \pm desvio-padrão das taxas de decomposição da celulose, hemicelulose e lignina após a $3^{\mathrm{a}}$ e $4^{\mathrm{a}}$ socas, e após o período acumulado das duas socas, em área com recolhimento variável de palha de cana-de-açúcar ${ }^{(1)}$.

\begin{tabular}{|c|c|c|c|c|c|c|c|c|c|}
\hline \multirow[t]{2}{*}{ Tratamentos $^{(2)}$} & \multicolumn{3}{|c|}{ Celulose (\%) } & \multicolumn{3}{|c|}{ Hemicelulose (\%) } & \multicolumn{3}{|c|}{ Lignina (\%) } \\
\hline & $3^{\mathrm{a}}$ soca & $4^{\mathrm{a}}$ soca & AC $3^{\mathrm{a}+4^{\mathrm{a}} \text { socas }}$ & $3^{\mathrm{a}}$ soca & $4^{\mathrm{a}}$ soca & AC $3^{\mathrm{a}+4^{\mathrm{a}} \text { socas }}$ & $3^{\mathrm{a}}$ soca & $4^{\mathrm{a}}$ soca & AC $3^{\mathrm{a}}+4^{\mathrm{a}}$ socas \\
\hline $100 \%$ & $80,0 \pm 3,0^{1}$ & $16,2 \pm 3,2$ & $96,2 \pm 0,6$ & $85,6 \pm 3,9$ & $10,7 \pm 3,8$ & $96,3 \pm 0,3$ & $43,2 \pm 7,6$ & $40,3 \pm 6,0$ & $83,4 \pm 3,7$ \\
\hline $75 \%$ & $81,9 \pm 2,5$ & $14,7 \pm 2,4$ & $96,5 \pm 0,2$ & $87,0 \pm 3,3$ & $9,4 \pm 3,3$ & $96,4 \pm 0,3$ & $47,2 \pm 7,1$ & $38,0 \pm 6,7$ & $85,2 \pm 1,4$ \\
\hline $50 \%$ & $84,1 \pm 2,2$ & $9,7 \pm 2,5$ & $93,8 \pm 1,0$ & $84,8 \pm 2,9$ & $9,6 \pm 4,0$ & $94,4 \pm 1,3$ & $53,4 \pm 7,5$ & $25,4 \pm 8,5$ & $78,9 \pm 3,3$ \\
\hline $25 \%$ & $81,1 \pm 3,2$ & $14,4 \pm 4,4$ & $95,5 \pm 1,5$ & $83,7 \pm 3,3$ & $12,5 \pm 3,0$ & $96,2 \pm 0,8$ & $49,4 \pm 4,5$ & $38,5 \pm 6,6$ & $87,9 \pm 3,4$ \\
\hline Média & $81,8 \mathrm{~A}$ & $13,6 \mathrm{~B}$ & 95,5 & $85,3 \mathrm{~A}$ & $10,6 \mathrm{~B}$ & 95,9 & $48,3 \mathrm{~A}$ & $35,6 \mathrm{~A}$ & 83,9 \\
\hline Regressão & ns & ns & ns & ns & ns & ns & ns & $* *(3)$ & ns \\
\hline CV (\%) & 3,33 & 24,9 & 1,10 & 4,19 & 36,26 & 0,87 & 14,78 & 21,54 & 3,84 \\
\hline
\end{tabular}

${ }^{(1)}$ Médias seguidas de letras iguais não diferem pelo teste de Tukey a $1 \%$ de probabilidade. ${ }^{(2)} \mathrm{Os}$ tratamentos indicam a percentagem de palha que foi mantida no solo após a colheita: $100 \%, 11,3 \mathrm{Mg} \mathrm{ha}^{-1}$ de palha; $75 \%, 8,5 \mathrm{Mg} \mathrm{ha}^{-1} ; 50 \%, 5,7 \mathrm{Mg} \mathrm{ha}^{-1}$; e $25 \%, 2,8 \mathrm{Mg} \mathrm{ha}^{-1}$. ${ }^{\text {ns }} \mathrm{Não}$ significativo. **Significativo a $1 \%$ de probabilidade. ${ }^{(3)} \mathrm{y}=50,24-0,69 \mathrm{x}+0,006 \mathrm{x}^{2}, \mathrm{R}^{2}=0,53^{* *}$. 
decomposição do $\mathrm{C}$ e de imobilização do $\mathrm{N}$. $\mathrm{Na} 4^{\mathrm{a}}$ soca, os valores de $\mathrm{C} / \mathrm{N}$ foram inferiores aos da $3^{\mathrm{a}}$, em todos os tratamentos, indício de que o material remanescente de uma safra para outra encontra-se em uma forma mais estável, com relação $\mathrm{C} / \mathrm{N}$ próxima a 20, sem diferença entre os tratamentos.

Os resultados obtidos no tratamento com $100 \%(11,3$ $\mathrm{Mg} \mathrm{ha}^{-1}$ ) de manutenção da palha, no presente estudo, que apresentou relação $\mathrm{C} / \mathrm{N}$ de 36,8 , corroboram os de Abramo Filho (1995) que relatou relação $\mathrm{C} / \mathrm{N}$ de 33 com a manutenção de toda a palha $\left(15 \mathrm{Mg} \mathrm{ha}^{-1}\right)$. Fortes et al. (2012) verificaram $\mathrm{C} / \mathrm{N}$ de 24 , após duas socas, também próxima da observada na segunda soca (20) neste trabalho.

Observou-se correlação negativa e significativa entre taxa de biomassa degradada e relação $\mathrm{C} / \mathrm{N}$ da palha remanescente (Figura 2). Assim, os maiores valores de relação $\mathrm{C} / \mathrm{N}$ nos tratamentos com as menores quantidades de palha, na $3^{\mathrm{a}}$ soca (Figura 2 A), confirmam que a decomposição foi de fato menos intensa nesses tratamentos. Esses resultados estão de acordo com o senso comum na área, segundo o qual a intensidade de decomposição de resíduos é inversamente proporcional à sua relação $\mathrm{C} / \mathrm{N}$ e reflete o grau de mineralização desses resíduos (Melillo et al., 1982). Eles também reforçam a ideia de se utilizar a relação $\mathrm{C} / \mathrm{N}$ como indicadora do grau de decomposição da palha neste tipo de estudo, pois ela é capaz de detectar diferenças na taxa de mineralização de compostos em uma mesma soca, o que pode auxiliar a decisão sobre o quanto se pode retirar do resíduo, em função do que se espera que volte para o solo, na forma de ciclagem, neste período.

Neste estudo, do decaimento do conteúdo bioquímico, ou estrutural (celulose, hemicelulose e lignina), da palha (Tabela 2), observou-se que tanto a celulose quanto a hemicelulose não sofreram interação entre a quantidade de palha e as socas. As diferenças para celulose e hemicelulose foram detectadas apenas entre socas, com maiores valores na $3^{\mathrm{a}}$ soca. A hemicelulose foi o carboidrato com maior taxa de mineralização, de $85,5 \%$, na $3^{\mathrm{a}}$ soca, e $95,9 \%$, após as duas socas. A celulose veio em seguida, com valores de 81,8 e $95,5 \%$, respectivamente. Já a lignina, com concentração inicial de $10 \%$, se decompôs parcialmente entre as socas, com mineralização quadrática significativa apenas na $4^{\mathrm{a}}$ soca, em que os menores valores ocorreram próximos ao tratamento com $50 \%$ de palha. Como as taxas de mineralização da celulose e da hemicelulose não foram afetadas pelos níveis de palha remanescente, seus conteúdos não constituem bons indicadores do efeito dessa prática sobre a decomposição dos resíduos culturais, e são úteis apenas para acompanhar o processo ao longo do tempo. A lignina, no entanto, serve como indicador para detectar os efeitos dos níveis de palha remanescente sobre a decomposição; porém, sua avaliação somente deve ser feita com tempos superiores a uma soca.

As elevadas taxas de mineralização (Tabela 2), tanto da hemicelulose quanto da celulose, bem como sua independência do nível de palha remanescente, seguiram a mesma tendência das observadas por
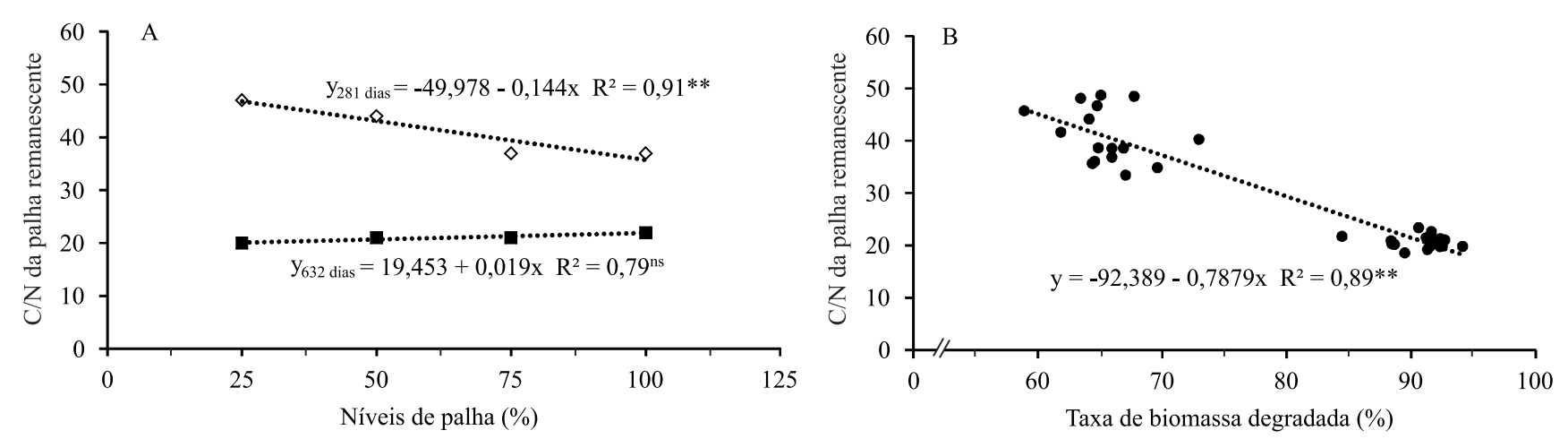

Figura 2. Relação $\mathrm{C} / \mathrm{N}$ da palha remanescente, ao final da $3^{\mathrm{a}}$ soca (281 dias), e no período acumulado das duas socas (632 dias) (A); além de correlação entre taxa de decomposição da biomassa e relação $\mathrm{C} / \mathrm{N}$ da palha (B), em área com recolhimento variável da palha de cana-de-açúcar. 
Dubeux et al. (2006), e concordam com a afirmação de que estes compostos são os mais facilmente decomponíveis na palha da cana-de-açúcar. Entretanto, Fortes et al. (2012) atribuem maior interferência da hemicelulose sobre o processo de decomposição inicial. Todos os trabalhos, porém, concordam que, em socas subsequentes, quando o resíduo permanece por mais tempo no campo, a lignina é o componente com maior interferência sobre o processo de decomposição da palha, uma vez que é um material reconhecidamente mais recalcitrante (Cotrufo et al., 2009).

A quantidade de carbono mineralizado aumentou linearmente com o aumento nos níveis de palha, tanto na $3^{\mathrm{a}}$ como na $4^{\mathrm{a}}$ socas (Figura 3 ). A manutenção de $100 \%$ de palha possibilitou mineralização de 3.720 $\mathrm{kg} \mathrm{ha}^{-1}$ de $\mathrm{C}$, enquanto sob manutenção de $25 \%$, esse valor não ultrapassou $914 \mathrm{~kg} \mathrm{ha}^{-1}$, após 281 dias de decomposição ( $3^{\mathrm{a}}$ soca). Já na $4^{\mathrm{a}}$ soca, os mesmos tratamentos mineralizaram, respectivamente, $1.303 \mathrm{e}$ $356 \mathrm{~kg} \mathrm{ha}^{-1} ;$ mesmo após duas socas para decomposição (Figura $3 \mathrm{~B}$ ). Assim, mesmo retirando-se o viés de trabalhar com taxas ao invés de valores absolutos, cabe destacar que diferentes quantidades de palha remanescente, mesmo com mineralização semelhante, contribuem com quantidades mineralizadas de $\mathrm{C}$ e $\mathrm{N}$ proporcionais ao seu aporte inicial. A qualidade do solo, portanto, também deve ser influenciada de forma proporcional a esses aportes.

Merece destaque o fato de que o potencial de mineralização do $\mathrm{N}$ aumentou linearmente com as quantidades de palha remanescente (Figura 3). Ainda que no primeiro ciclo as taxas não sejam elevadas (Tabela 1), após as duas socas, a mineralização foi de $40 \mathrm{~kg} \mathrm{ha}^{-1}$, no tratamento com manutenção de $100 \%$, e de $10 \mathrm{~kg} \mathrm{ha}^{-1}$, no com $25 \%$. Esses valores, apesar de sensivelmente inferiores aos de carbono, e insuficientes para suprir a demanda da cana-de-açúcar pelo nutriente, que está entre 1,0 e 1,2 kg por tonelada de colmo produzida (Vitti et al., 2008), podem contribuir para a redução parcial da necessidade de aplicação do insumo no longo prazo (Trivelin et al., 2013).

A grande questão a ser abordada, no entanto, diz respeito aos benefícios reais que a mineralização desses elementos podem trazer ao agrossistema da cana-deaçúcar, e também ao ambiente. Graham et al. (2002), Robertson \& Thorburn (2007), Galdos et al. (2009) e Trivelin et al. (2013) mencionam que o aumento no aporte de $\mathrm{C} \mathrm{e} \mathrm{N}$, fornecidos pela mineralização da palha,
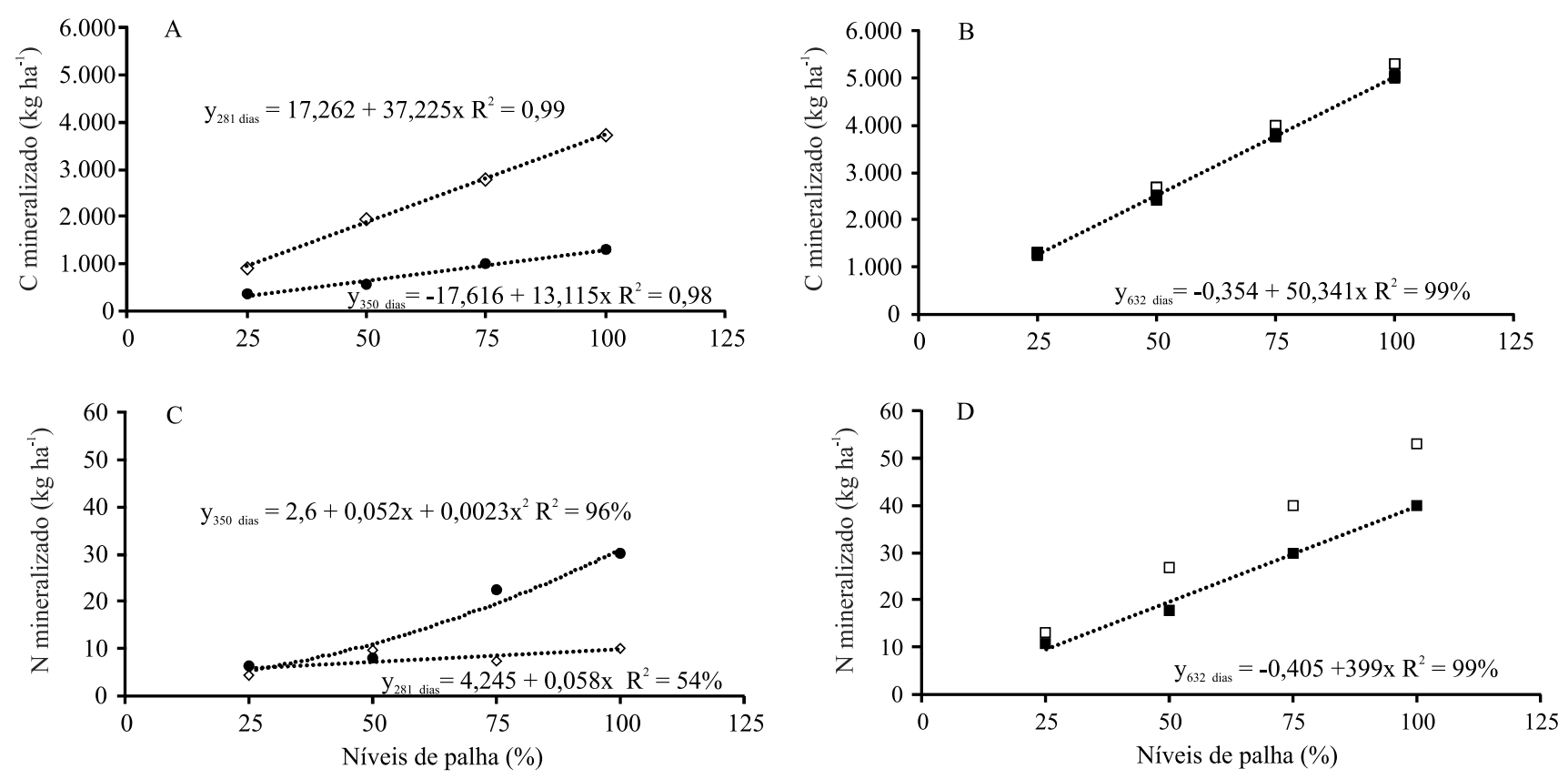

Figura 3. Quantidade de carbono (A) e nitrogênio (C) mineralizados a partir da palha, na $3^{\mathrm{a}}\left(281\right.$ dias) e $4^{\mathrm{a}}(350$ dias) socas; bem como total acumulado de carbono (B) e nitrogênio (D) nas 2 socas (632 dias), em com recolhimento variável da palha da cana-de-açúcar. 
melhora a estabilidade de agregados, incrementa os teores de matéria orgânica e os estoques de carbono e nitrogênio no solo, e reduzem a erosão e a necessidade de aporte de fertilizantes. Assim, os resultados do presente estudo apontam para necessidade de cautela antes de uma decisão efetiva quanto ao recolhimento da palha de cana-de-açúcar, no sentido de se evitar a diminuição da qualidade do solo a longo prazo.

\section{Conclusões}

1. O recolhimento de quantidade variável de palha da cana-de-açúcar não altera as taxas de decomposição da biomassa, nem a mineralização do carbono, da celulose, da hemicelulose e da lignina, após uma soca.

2. O processo de decomposição da palha não se esgota mesmo após duas socas, independentemente da quantidade de resíduo mantida sobre o solo.

3. A relação $\mathrm{C} / \mathrm{N}$ e a decomposição da lignina servem como indicadores para avaliar os efeitos dos níveis de retirada de palha sobre a decomposição dos resíduos culturais.

4. Em termos absolutos, quanto menor a retirada da palha do campo, maior a quantidade de carbono e nitrogênio mineralizados, mesmo que não haja diferenças na taxa de decomposição.

\section{Agradecimentos}

À Empresa Brasileira de Pesquisa Agropecuária (Embrapa, projetos números 0210000080000 e 021107022000100) e à Petrobrás, pelo apoio financeiro.

\section{Referências}

ABRAMO FILHO, J. Decomposição da palha de cana-deaçúcar em canavial colhido sem queima, mecanicamente. 1995. 91p. Dissertação (Mestrado) - Universidade Estadual Paulista, Rio Claro.

AQUINO, G.S. de. Desenvolvimento e produção de canade-açúcar em sistema sob palhada. 2012. 86p. Dissertação (Mestrado) - Universidade Estadual de Londrina, Londrina.

BOCOCK, K.L.; GILBERT, O.J.W. The disappearance of leaf litter under different woodland conditions. Plant and Soil, v.9, p.179185, 1957. DOI: 10.1007/BF01398924.

COTRUFO, F.C.; GALDO, I.D.; PIERMATTEO, D. Litter decomposition: concepts, methods and future perspectives. In: KUTSCH, W.L.; BAHN, M.; HEINEMEYER, A. (Ed.). Soil carbon dynamics: an integrated methodology. Cambridge: Cambridge University, 2009. p.76-90.
DUBEUX, J.C.B.; SOLLENBERGER, L.E.; INTERRANTE, S.M.; VENDRAMINI, J.M.B.; STEWART, R.L. Litter decomposition and mineralization in Bahiagrass pastures managed at different intensities. Crop Science, v.46, p.1305-1310, 2006. DOI: 10.2135/ cropsci2005.08-0263.

FAO. FOOD AND AGRICULTURE ORGANIZATION OF THE UNITED NATIONS. FAOSTAT: food and agricultural commodities production. 2014. Disponível em: $<$ http://faostat.fao. org/site/339/default.aspx $\geq$. Acesso em: 10 maio 2015.

FORTES, C.; TRIVELIN, P.C.O.; VITTI, A.C. Long-term decomposition of sugarcane harvest residues in Sao Paulo state, Brazil. Biomass and Bionergy, v.42, p.189-198, 2012. DOI: 10.1016/j.biombioe.2012.03.011.

GALDOS, M.V.; CERRI, C.C.; CERRI, C.E.P.; PAUSTIAN, K.; VAN ANTWERPEN, R. Simulation of soil carbon dynamics under sugarcane with the CENTURY Model. Soil Science Society of America Journal, v.73, p.802-811, 2009. DOI: 10.2136/ sssaj2007.0285.

GALDOS, M.V.; CERRI, C.C.; CERRI, C.E.P.; PAUSTIAN, K.; VAN ANTWERPEN, R. Simulation of sugarcane residue decomposition and aboveground growth. Plant and Soil, v.326, p.243-259, 2010. DOI: 10.1007/s11104-009-0004-3.

GRAHAM, M.H.; HAYNES, R.J.; MEYER, J.H. Changes in soil chemistry and aggregate stability induced by fertilizer applications, burning and trash retention on a long-term sugarcane experiment in South Africa. European Journal of Soil Science, v.53, p.589-598, 2002. DOI: 10.1046/j.1365-2389.2002.00472.x.

IVO, W.M.P. de M.; SILVA, E.F. da; SILVA, P.A.; SANTIAGO, A.D.; MARAFON, A.C. Impacto do manejo da palhada da canade-açúcar na produção e decomposição desses resíduos. In: CONGRESSO BRASILEIRO DE CIÊNCIA DO SOLO, 34., 2013. Florianópolis. Ciência do solo: para quê e para quem: resumos expandidos. Florianópolis: Sociedade Brasileira de Ciência do Solo, 2013. 4p. Disponível em: <http://www.eventossolos.org.br/ cbcs2013/>. Acesso em: 10 maio 2015.

LEAL, M.R.L.V.; GALDOS, M.V.; SCARPARE, F.V.; SEABRA, J.E.A.; WALTER, A.; OLIVEIRA, C.O.F. Sugarcane straw availability, quality, recovery and energy use: a literature review. Biomass and Bionergy, v.53, p.11-19, 2013. DOI: 10.1016/j. biombioe.2013.03.007.

LEITE, R.C. de C. (Coord.). Bioetanol combustível: uma oportunidade para o Brasil. Brasília: Centro de Gestão e Estudos Estratégicos, 2009. 536p. Disponível em: $<$ http://www.cogen.com. br/paper/2010/Livro_Bioetanol_Cana_Acucar_2009.pdf $>$. Acesso em: 10 jun. 2015.

MELILLO, J.M.; ABER, J.D.; MURATORE, J.F. Nitrogen and lignin control of hardwood leaf litter decomposition dynamics. Ecology, v.63, p.621-626, 1982. DOI: 10.2307/1936780.

PERES, J.G.; SOUZA, C.F.; LAVORENTI, N.A. Avaliação dos efeitos da cobertura de palha de cana-de-açúcar na umidade e perda de água do solo. Engenharia Agrícola, v.30, p.875-886, 2010. DOI: $10.1590 / \mathrm{S} 0100-69162010000500010$.

ROBERTSON, F.A.; THORBURN, P.J. Decomposition of sugarcane harvest residue in different climatic zones. Australian

Pesq. agropec. bras., Brasília, v.51, n.9, p.1492-1500, set. 2016 DOI: 10.1590/S0100-204X2016000900048 
Journal of Soil Research, v.45, p.1-11, 2007. DOI: 10.1071/ SR06079.

ROLIM, G. de S.; CAMARGO, M.B.P. de; LANIA, D.G.; MORAES, J.F.L. de. Classificação climática de Köppen e de Thornthwaite e sua aplicabilidade na determinação de zonas agroclimáticas para o Estado de São Paulo. Bragantia, v.66, p.711720, 2007. DOI: 10.1590/S0006-87052007000400022.

ROSSETTO, R.; VITTI, A.C.; GAVA, G.J.C.; MELLIS E.V.; VARGAS V.P.; CANTARELLA, H.; PRADO, H. do; DIAS, F.L.F.; LANDELL, M.G.A.; BRANCALIÃO, S.R.; GARCIA J.C. Cana-de-açúcar: cultivo com sustentabilidade. Informações Agronômicas, n.124, p.8-13, 2008.

SANTOS, H.G. dos; JACOMINE, P.K.T.; ANJOS, L.H.C. dos; OLIVEIRA, V.A. de; OLIVEIRA, J.B. de; COELHO, M.R.; LUMBRERAS, J.F.; CUNHA, T.J.F. da (Ed.). Sistema brasileiro de classificação dos solos. 2.ed. Rio de Janeiro: Embrapa Solos, 2006. 306p.

SOUSA, G.B.; MARTINS FILHO, M.V.; MATIAS, S.S.R. Perda de solo, matéria orgânica e nutrientes por erosão hídrica em uma vertente coberta com diferentes quantidades de palha de cana-deaçúcar em Guariba-SP. Engenharia Agrícola, v.32, p.490-500, 2012. DOI: 10.1590/S0100-69162012000300008.
THOMAS, R.J.; ASAKAWA, N.M. Decomposition of leaf litter from tropical forage grasses and legumes. Soil Biology and Biochemistry, v.25, p.1351-1361, 1993. DOI: 10.1016/00380717(93)90050-L.

TRIVELIN, P.C.O.; FRANCO, H.C.J.; OTTO, R.; FERREIRA, D.A.; VITTI, A.C.; FORTES, C.; FARONI, C.E.; OLIVEIRA, E.C.A.; CANTARELLA, H. Impact of sugarcane trash on fertilizer requirements for São Paulo, Brazil. Scientia Agricola, v.70, p.345352, 2013. DOI: 10.1590/S0103-90162013000500009.

VAN SOEST, P.J.; ROBERTSON, J.B.; LEWIS, B.A. Methods for dietary fiber, neutral detergent fiber, and nonstarch polysaccharides in relation to animal nutrition. Journal of Dairy Science, v.74, p.3583-3597, 1991. DOI: 10.3168/jds.S0022-0302(91)78551-2.

VITTI, A.C.; TRIVELIN, P.C.O.; CANTARELLA, H.; FARONI, C.E.; FRANCO, H.C.J.; OTTO, R.; TRIVELIN, M.O.; TOALIARI, J.G. Mineralização da palhada e desenvolvimento de raízes de cana-de-açúcar relacionados à adubação nitrogenada de plantio. Revista Brasileira de Ciência do Solo, v.32, p.2757-2762, 2008. DOI: 10.1590/S0100-06832008000700020.

ZHANG, D.; HUI, D.; LUO, Y.; ZHOU, G. Rates of litter decomposition in terrestrial ecosystems: global patterns and controlling factors. Journal of Plant Ecology, v.1, p.85-93, 2008. DOI: $10.1093 /$ jpe/rtn002.

Recebido em 30 de julho de 2015 e aprovado em 2 de dezembro de 2015 